NOUVELLE

\section{Un modèle avancé de cellule synthétique minimale}

Nicolas Puff ${ }^{1,2}$
> Créer artificiellement des cellules qui possèdent les propriétés minimales de la vie : voilà une tâche ambitieuse que de nombreuses équipes de recherche à travers le monde se sont fixées [1-3]. Les différentes approches envisagées - prébiotique, semisynthétique ou supramoléculaire - se basent toutes sur un même constat : même le plus simple organisme unicellulaire existant aujourd'hui est extrêmement complexe et possède une telle diversité et richesse de comportements que tout modèle ne peut être que très approximatif. Cependant, cette complexité est-elle vraiment nécessaire à la «vie » de la cellule ou est-il possible en laboratoire de construire quelque chose de beaucoup plus simple, à partir d'un nombre limité de composants, ayant les caractéristiques de la vie? Cette démarche bottom-up (partir d'éléments simples pour construire un système dynamique complexe), utilisée pour l'élaboration de ces systèmes chimiques « autopoïétiques » [4] nécessite au préalable de définir ce que l'on entend par «vie ». Il est communément accepté aujourd'hui qu'un assemblage moléculaire local est « vivant » si : (1) il est capable de se régénérer ; (2) il peut s'autoreproduire ; (3) il est capable d'évolution. Trois composants critiques

Composants principaux du système

\begin{tabular}{|c|c|c|c|c|}
\hline amomers & ADN & 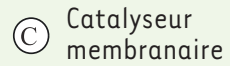 & Onico & $\begin{array}{l}\text { Précurseur membranaire } \\
\text { cationique }\end{array}$ \\
\hline G & Polymérase & Marqueur & ONo & Lipide cationique \\
\hline & Phospholipides & & 0 & Électrolyte \\
\hline
\end{tabular}

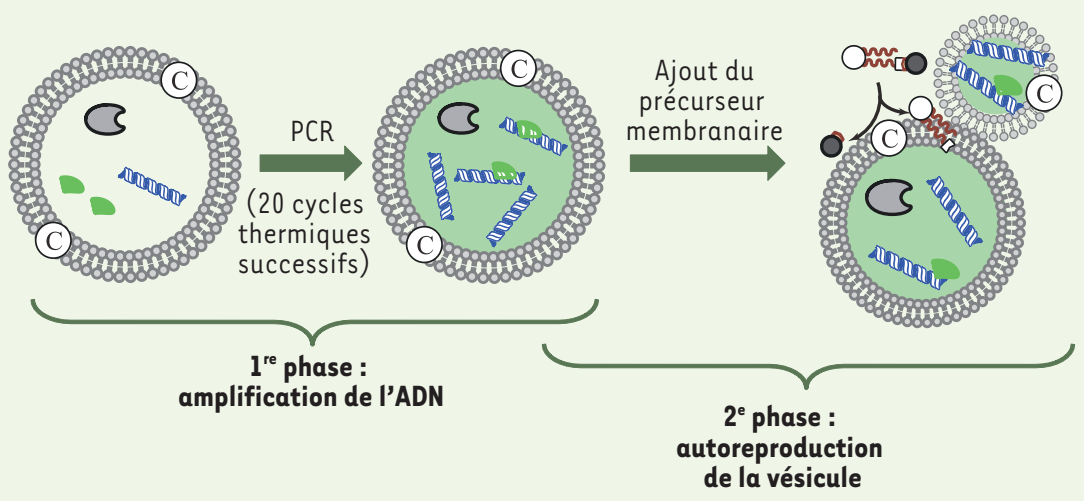

Figure 1. Représentation schématique du mécanisme d'autoreproduction des vésicules lié à l'amplification de l'ADN encapsulé. L'assemblage des différents composants du système produit des vésicules contenant de l'ADN et les éléments nécessaires à sa réplication (polymérase, désoxyribonucléotides et amorces spécifiques constituées d'oligonucléotides). L'ADN est ensuite amplifié au sein du compartiment par PCR (20 cycles thermiques successifs : $94^{\circ} \mathrm{C}$ pendant $15 \mathrm{~s}$ suivi de $90 \mathrm{~s} \mathrm{à} 68^{\circ} \mathrm{C}$ ). Le précurseur lipidique est ensuite ajouté à la solution et sera converti par catalyse en une molécule membranaire. Cette dernière étape induit la croissance de la membrane et finalement conduit à la séparation de la vésicule fille de la vésicule initiale.
${ }^{1}$ Université Pierre et Marie Curie (Paris 6), UFR 925, département de physique,

Paris, France ;

2 matière et systèmes complexes (MSC), UMR 7057 CNRS et université Paris Diderot (Paris 7), 10 , rue A. Domon et L. Duquet,

case courrier 7056,

75205 Paris Cedex 13, France. nicolas.puff@upmc.fr

nécessaires à l'élaboration d'une telle cellule minimale ont été identifiés [2] : une substance porteuse de l'information génétique ( $A R N$ ou $A D N$ ), un catalyseur et un compartiment clos semiperméable (une vésicule par exemple).

Autoreproduction du compartiment lié à l'amplification de l'ADN encapsulé Dans une publication récente de Nature Chemistry [5], Tadashi Sugawara et al. décrivent comment la réplication de I'ADN au sein d'un compartiment lipidique (une vésicule géante unilamellaire) peut induire la croissance puis la division du compartiment. Lors de travaux antérieurs, ces mêmes auteurs avaient déjà décrit ces deux phénomènes mais de manière indépendante. D'une part, l'amplification par PCR d'un fragment d'ADN au sein d'une vésicule géante phospholipidique a été réalisée avec succès [6]. D'autre part, il a été montré que sous certaines conditions, des vésicules géantes (d'une taille d'une dizaine de micromètres et par conséquent visualisables directement en microscopie optique) étaient capables de croître puis de se diviser [7]. Un catalyseur amphiphile présent dans la membrane des vésicules permet en effet la conversion, par clivage, des précurseurs lipidiques ajoutés à la solution de vésicules en lipides membranaires, ces derniers conduisant à la croissance puis à la division de la vésicule initiale.

Le tour de force actuel réside dans le fait d'avoir rendu possible la réalisation de ces deux phénomènes au sein d'un même système supramoléculaire. Dans un premier temps, l'ADN est amplifié (Figure 1). Cette amplification est préalable à l'ajout du précurseur 


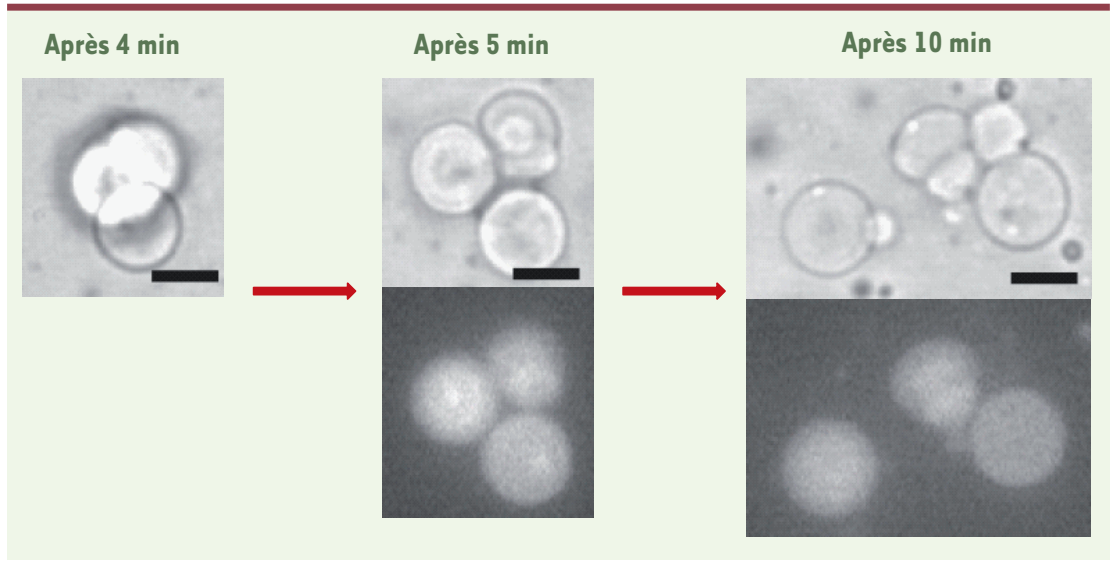

Figure 2. Observation en temps réel après ajout du précurseur membranaire des changements morphologiques des vésicules contenant l'ADN amplifié. Les vésicules commencent à croître et à se diviser quelques minutes seulement après l'ajout du précurseur membranaire. La division complète de la vésicule initiale en quatre vésicules se produit après environ six minutes; les vésicules finissent par se séparer (série d'images du haut). L'ADN est effectivement partagé entre les différentes vésicules filles (détection en microscopie de fluorescence ; série d'images du bas). Barre d'échelle : $10 \mu \mathrm{m}$.

membranaire car les vésicules issues des divisions successives doivent aussi contenir de I'ADN. Dans un second temps, le précurseur membranaire cationique est ajouté à la solution de vésicules, conduisant in fine à l'incorporation de nouveaux lipides cationiques dans la membrane. Par rapport au travail précédemment cité [7] où seuls le lipide cationique, son précurseur et le catalyseur amphiphile étaient présents, la composition moléculaire de la membrane vésiculaire a été spécifiquement élaborée pour permettre la réplication de I'ADN tout en conservant les propriétés d'autoreproduction vésiculaire. Ainsi, des lipides zwitterioniques ( $P O P C$ ) et anioniques (POPG) ont été ajoutés d'une part pour que la cavité centrale de la vésicule puisse contenir une solution saline simple, et d'autre part pour réduire la charge de surface permettant ainsi une meilleure stabilité vis-à-vis des changements de température et de force ionique du milieu inhérents à la PCR. L'ADN polyanionique interagit fortement avec les lipides cationiques nouvellement incorporés dans la membrane vésiculaire. Ceci conduit alors, du fait de l'augmentation de la concentration en ADN dans la vésicule, à accélérer l'incorporation de nouveaux lipides dans la membrane et donc la croissance et la division des vésicules (Figure 1). La croissance et les changements morphologiques des vésicules se produisent dans les quelques minutes qui suivent l'addition du précurseur membranaire. De multiples vésicules de taille équivalente à la vésicule initiale et contenant de I'ADN sont produites en moins d'une quinzaine de minutes (Figure 2).

\section{Une synergie prometteuse}

En présence d'ADN amplifié, la fréquence de division des vésicules est nettement plus grande qu'en son absence. Sugawara et al. démontrent que l'efficacité avec laquelle les vésicules croissent et se divisent est en effet dépendante de la quantité d'ADN produite dans les vésicules, elle-même fixée par le nombre de cycles de PCR. Cette synergie résulte d'une série d'interactions moléculaires complexes au niveau de la membrane entre I'ADN fraîchement produit, les lipides membranaires, le catalyseur amphiphile et le précurseur lipidique cationique. Pour expliquer ce comportement, les auteurs proposent un mécanisme (en partie spéculatif) basé sur l'accumulation locale de lipides membranaires cationiques autour de zones de la membrane interne riches en ADN polyanionique conduisant, à terme, à son enfouissement dans la membrane. Cet état transitoire facilite alors localement une incorporation supplémentaire du précurseur cationique et aboutit à un déséquilibre du nombre de molécules entre les deux feuillets lipidiques de la membrane qui se traduit finalement au niveau vésiculaire en une préstructure (bourgeonnement) propice à la division. Ce système, où l'ADN amplifié participe à la reproduction de son compartiment sans action de protéines spécifiques, diffère à ce niveau des cellules qui, elles, se divisent en connectant physiquement $A D N$ et membrane via des protéines spécifiques. II apporte cependant un éclairage précieux sur ce que pouvaient être ces processus dans les systèmes prébiotiques.

\section{Un monde encore à explorer}

Ce travail est une preuve de principe qu'un système dynamique complexe possédant certaines caractéristiques de la vie (autoreproduction principalement) peut être artificiellement construit en exploitant réactions chimiques et principe d'auto-organisation. Malgré son comportement exceptionnel et la chimie spécifique élégamment développée pour l'élaborer, ce système présente encore à ce stade certaines limites. D'une part, lors des évènements successifs d'autoreproduction des vésicules, la composition de la membrane change - elle s'appauvrit en phospholipides - conduisant à une reproduction vésiculaire de facto limitée. II faut également noter que dans ce système, les catalyseurs (la polymérase et le catalyseur de la synthèse de surfactant) ne sont pas produits in situ au contraire de I'ADN et du lipide cationique. Cela conduit fatalement, après un certain nombre 
d'autoreproductions vésiculaires, à une population de compartiments non actifs. Pour surmonter ces limites, les auteurs envisagent de fusionner avec les vésicules filles des vésicules contenant les ingrédients nécessaires au maintien de l'activité du système. D'autres approches sont parallèlement élaborées dans le but d'obtenir une production de l'ensemble des constituants du système lors d'un cycle sans apport de molécules additionnelles. Dans ce cadre, l'intégration d'ARN catalytiques autoréplicateurs (les ribozymes) au sein de vésicules lipidiques autoreproductrices représente également l'une des voies possibles vers la synthèse artificielle de la vie [2]. La construction d'une cellule synthétique minimale est en tous cas un défi passionnant, à l'interface de multiples champs (biologie synthétique, étude de l'origine de la vie ) et dont l'issue, malgré le long chemin restant à parcourir, paraît aujourd'hui envisageable. $\diamond$

\section{An advanced model \\ of a minimalistic synthetic cell}

\section{CONFLIT D'INTERÊTS}

L'auteur déclare n'avoir aucun conflit d'intérêts concernant les données publiées dans cet article.

\section{REMERCIEMENTS}

L'auteur remercie Michel Seigneuret pour sa relecture du manuscrit.

\section{RéFÉRENCES}

1. Luisi PL, Ferri F, Stano P. Approaches to semi-synthetic minimal cells: a review. Die Naturwissenschaften $2006 ; 93: 1-13$.

2. Szostak JW, Bartel DP, Luisi PL. Synthesizing life. Nature 2001 ; 409 : 387-90.

3. Walde P. Building artificial cells and protocell models: experimental approaches with lipid vesicles. Bioessays $2010 ; 32: 296-303$.

4. Varela FG, Maturana HR, Uribe R. Autopoiesis: the organization of living systems, its characterization and a model. Curr Modern Biol 1974 ; 5 : 187-96.

5. Kurihara K, Tamura M, Shohda K, et al. Selfreproduction of supramolecular giant vesicles combined with the amplification of encapsulated DNA. Nat Chem $2011 ; 3$ : 775-81.

6. Shohda KI, Tamura M, Kageyama Y, et al. Compartment size dependence of performance of polymerase chain reaction inside giant vesicles. Soft Matter 2011; 7 : 3750-3.

7. Takakura K, Sugawara T. Membrane dynamics of a myelin-like giant multilamellar vesicle applicable to a self-reproducing system. Langmuir 2004 ; 20 : 3832-4.

\section{NOUVELLE}

\section{Traitement de la détresse respiratoire des prématurés}

Un agoniste de la voie de signalisation hedgehog prévient les effets néfastes des glucocorticoïdes

Amélie Griveau ${ }^{1,2}$

\author{
${ }^{1}$ Department of Pediatrics, Division of Neonatology, \\ ${ }^{2}$ Howard Hughes Medical Institute, Eli and Edythe Broad \\ Institute for Stem Cell Research and Regeneration Medicine, \\ University of California San Francisco, San Francisco, \\ 35 Medical Center Way - RMB 932D, CA 94143, États-Unis. \\ GriveauA@stemcell.ucsf.edu
}

\begin{abstract}
Administration des glucocorticoïdes chez les prématurés: bénéfice et risques Les glucocorticoïdes (GC) sont des hormones stéroïdes sécrétées en faible quantité par la glande surrénale. Une modification de leur taux peut conduire à des altérations importantes du développement et de la maturation de certains organes tels que les poumons ou le cerveau. Divers GC synthétiques comme la dexaméthasone, la prednisolone, la $\beta$-méthasone ou encore l'hydrocortisone, ont été développés et administrés en période anté- ou postnatale afin de prévenir ou de traiter le risque de détresse respiratoire ainsi que d'autres complications souvent rencontrées chez les prématurés $[1,10]$. Les pathologies pulmonaires sont principalement liées à un défaut de production des protéines

du surfactant : cet « enduit alvéolaire » de composition complexe, très riche en phospholipides, empêche les alvéoles de se collaber lors de l'expiration, grâce à ses propriétés tensioactives. L'administration de GC aux femmes à risque ou aux enfants prématurés permet, entre autres, de réduire l'inflammation et de stimuler la synthèse des protéines du surfactant par les pneumocytes de type II. Malgré ces effets bénéfiques, de plus en plus d'études indiquent que l'administration périnatale de GC à des doses supraphysiologiques entraîne des déficits cognitifs ou moteurs à long terme liés à une augmentation de l'incidence des lésions de la substance blanche et de l'infirmité motrice cérébrale, ou encore à des défauts de développement du cervelet $[2-4,10]$.
\end{abstract}

Antagonisme entre les glucocorticoïdes et la voie Shh pour la croissance du cervelet

Le cervelet est impliqué dans la coordination motrice et le relais d'informations vers le cortex cérébral chez l'adulte. Cette structure grossit considérablement pendant la période périnatale via l'expansion des précurseurs des neurones granulaires cérébelleux (PNGC), induite par la voie de signalisation hedgehog [5]. Sonic hedgehog (Shh), une protéine sécrétée par les cellules de Purkinje, en se fixant sur son récepteur transmembranaire Patched (Ptch) présent au niveau des PNGC, entraîne la dérépression de Smoothened (Smo). II en résulte une activation de la cascade de signalisation intracellulaire, dont en particulier Glil, 